\title{
Diz Eklemi Momentinin Tahmini İçin Makine Öğrenmesi Yöntemlerinin İncelenmesi
}

\author{
*1 Kasım Serbest and ${ }^{1}$ Serhan Ayberk Kilıç \\ ${ }^{1}$ Sakarya Uygulamalı Bilimler Üniversitesi, Teknoloji Fakültesi, Mekatronik Mühendisliği Bölümü, Türkiye
}

\begin{abstract}
Özet
İnsan uzuvlarında meydana gelen eklem momentlerinin belirlenmesi değişik uygulamalar için önemlidir. Önceki çalışmalarda eklem momentleri matematiksel yöntemler, bu alana özgü yazılımlar veya yapay zeka yöntemleri ile belirlenmiştir. Yapay zeka teknikleri ile yapılan tahminlerde çoğunlukla elektromiyografi (EMG) sinyalinden faydalanılmaktadır. Ancak EMG ile çalışmanın bazı dezavantajları bulunmaktadır. $\mathrm{Bu}$ çalışmada kütle ve atalet özelliklerine dayalı olarak elde edilen veriler ile farklı makine öğrenmesi algoritmaları kullanılarak diz eklemi momenti tahmin edilmiştir. Oturup kalkma hareketinin analizine yönelik yapılan klinik çalışmamıza 10 adet sağlıklı denek katılmıştır. Deneklerden elde edilen veriler doğrusal regresyon, destek vektör makineleri, polinom regresyonu, rassal orman, XGboost, Light GBM ve yapay sinir ağı algoritmalarının eğitiminde kullanılmıştır. Sonuçlara bakıldığında diz eklemi momentini tahmin etmede en yüksek başarı XGBoost algoritmasında (\%94.0), en düşük başarı ise destek vektör makineleri algoritmasında (\%71.5) elde edilmiştir.
\end{abstract}

Anahtar kelimeler: Biyomekanik analiz, oturup kalkma, hareket analizi, MATLAB

\section{Analysis of Machine Learning Techniques for Estimation of Knee Joint Moment}

\begin{abstract}
Determining joint moment in human limbs is important for various applications. In previous studies, joint moments were determined using mathematical methods, specific software or artificial intelligence techniques. The electromyography (EMG) is usually used in predictions with artificial intelligence algorithm. However, EMG signal has some disadvantages. In this study, knee joint moment was estimated using different machine learning algorithm based on mass and inertia properties of the limbs. Ten unimpaired subjects participated the clinical study to analyze sit-to-stand movement. The data of subjects were used to train linear regression, support vector machine, polynomial regression, random forest, XGboost, Light GBM and artificial neural network algorithms. According the results, XGBoost algorithm has a highest level of accuracy $(94.0 \%)$ and support vector machine algorithm has a lowest level of accuracy $(71.5 \%)$.
\end{abstract}

Key words: Biomechanical analysis, sit-to-stand, motion analysis, MATLAB

\section{Giriş}

İnsan uzuvlarındaki eklem momentlerinin belirlenmesi sportif faaliyetler, protez ve ortez tasarımları, ergonomik çalışmalar, insan-makine etkileşimi ve rehabilitasyon teknikleri açısından

*Corresponding author: Address: Faculty of Technology, Department of Mechatronics Engineering Sakarya University of Applied Sciences, TURKEY. E-mail address: kserbest@ subu.edu.tr, Phone: +902646160305 
önem arz etmektedir. Eklem momentleri geleneksel olarak açık zincir mekanik yapıya sahip eklemuzuv modelleri yardımıyla hesaplanmaktadır [1]. Açısal momentumun korunumu prensibine göre analiz edilen bu modeller çoğunlukla ters dinamik yaklaşım [2] ile çözülmektedir. Ters dinamik yaklaşımda zorlayıcı denklemlerle uğraşmak gerekmektedir.

Eklem momentlerinin hesaplanmasında kullanılan bir diğer yaklaşım simülasyon yazılımlarından faydalanmaktır. Bunlar Anybody, OpenSim [3] ve LifeModeler [4] gibi kas-iskelet sistemi analizleri için özel geliştirilmiş yazılımların yanı sıra MATLAB [5] ve ADAMS [6] gibi genel amaçlı yazımlar olabilmektedir. Bu yazımları kullanarak eklem momentlerini analiz etmek özel bir uzmanlık gerektirmektedir.

Son zamanlarda eklem momentlerinin belirlenmesinde yapay zeka teknikleri de tercih edilmektedir [7]. Farklı çalışmalarda bulanık mantık, yapay sinir ağları, nöro-bulanık mantık ve lojistik regresyon gibi yöntemlerin kullanıldığı görülmektedir. Bununla birlikte doğrusal regresyon, karar ağac1, destek vektör makineleri ve rassal orman gibi makine öğrenmesi algoritmaları da tercih edilmektedir. Makine öğrenmesi algoritmalarının eğitimi için en çok tercih edilen veri elektromiyografidir (EMG). Kas kasılması sırasında meydana gelen EMG sinyali, eklem momentlerinin yapay zeka yöntemleri ile tahmininde en temel eğitim verisi olarak kullanılmaktadır [8]. Ancak EMG verisi ile çalışırken bazı zorluklarla karşılaşmak mümkündür. Bunlardan bazıları; elektrot yerleşimi, sinyalin kalibrasyonu, diğer kaslardan gelen gürültü ve sinyalin kişiye özgü farklılıklar içermesi şeklinde sıralanabilir.

Bu çalışmanın amacı insan uzuvlarındaki eklem momentlerinin tahmini için kinematik ve kütle özelliklerine dayalı farklı makine öğrenmesi algoritmalarının incelenmesidir. Bu doğrultuda oturup kalkma hareketine yönelik bir klinik çalışma gerçekleştirilmiş ve katılımcıların hareket analizi verileri kullanılarak MATLAB ortamında diz eklemi momenti hesaplanmıştır. Ardından eklem momenti, katılımcıların kütle özellikleri ve diz ekleminin kinematik verileri doğrusal regresyon (DR), destek vektör makineleri (DVM), polinom regresyonu (PR), rassal orman (RO), XGBoost (XGB), Light GBM (LGM) ve yapay sinir ağları algoritmalarının (YSA) eğitiminde kullanılmıştır. Elde edilen sonuçlar özellikle XGBoost ve LGM algoritmalarının eklem momentlerini tahmin etmede daha başarılı olduğunu göstermiştir.

\section{Yöntem}

$\mathrm{Bu}$ çalışma günlük aktiviteler sırasında en çok sergilenen hareketlerden biri olan oturup kalkma

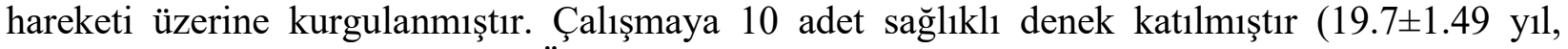
$72.23 \pm 15.20 \mathrm{~kg}, 174 \pm 7.19 \mathrm{~cm})$. Öncelikle oturup kalkma eyleminin hareket analizi yapılmıştır. Daha sonra hareket verileri ve kütle özellikleri kullanılarak MATLAB ortamında hareketin benzetimi yapılmış ve diz eklemi momenti hesaplanmıştır. Daha sonra hesaplanan moment ve katılımcıların kütle özellikleri kullanılarak farklı makine öğrenmesi algoritmaları eğitilmiştir. İlerleyen bölümlerde bu hususların detayları yer almaktadır. 


\subsection{Eklem-uzuv modeli}

Oturup kalkma hareketinin analizine uygun şekilde vücudun median düzleme göre yarısı referans alınarak alt bacak, üst bacak ve gövde uzuvları ile ayak bileği, diz ve kalça eklemlerinden oluşan bir eklem-uzuv modeli oluşturulmuştur (Şekil 1). Ayak bileği zemine sabitlenmiş bir mafsal olarak modellenmiştir. Diz ve kalça eklemleri tek serbestlik dereceli döner mafsal, uzuvlar ise katı cisim olarak temsil edilmiştir. Uzuvlara ait kütle, uzunluk, kütle merkezi ve atalet momenti verileri önceki çalışmalardan adapte edilmiştir [9].

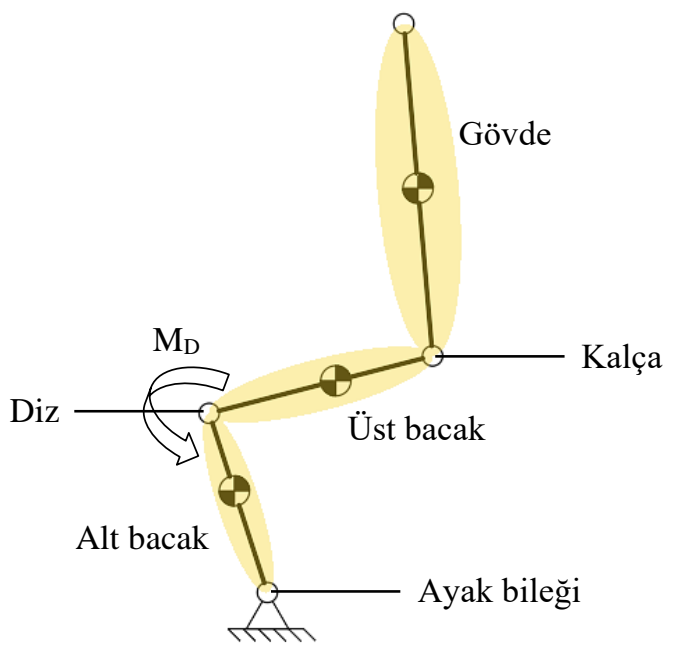

Şekil 1. Eklem-uzuv modeli. $\mathrm{M}_{\mathrm{D}}$ : Diz eklemi momenti

\subsection{Hareket analizi}

Oturup kalkma hareketinin analizi için katılımcıların ayak bileği, diz, kalça ve omuz bölgelerine pasif yansıtıcı işaretleyiciler yerleştirilmiştir. Katılımcılar sırt desteği olmayan bir tabure üzerinden kendi belirledikleri bir hızda oturup kalkma hareketini gerçekleştirmişlerdir. Bu esnada görüntüler sagital düzleme yerleştirilen bir dijital kamera (30 saniye/kare) ile kaydedilmiştir. Tracker [10] video analiz programı vasıtasıyla görüntülerdeki işaretleyicilerin konum verileri tespit edilmiştir. Ardından diz ve kalça eklemine ait kinematik (açısal yer değiştirme, hız ve ivme) veriler elde edilmiştir. Bu esnada hareket analizi verilere alçak geçiren filtre uygulanarak hareketin doğasına uygun olmayan saç1lmalar önlenmiştir.

\subsection{Eklem momentlerinin hesaplanması}

Eklem momentlerinin hesaplanması için Şekil 1'de görülen eklem-uzuv modeli MATLAB Simscape Multibody ortamında oluşturulmuştur. Şekil 2'de dört uzuv ve üç mafsaldan oluşan eklem-uzuv modelinin MATLAB Multibody blok diyagramlarından oluşan yapısı görülmektedir. Burada hareket analizi sonucunda hesaplanan kinematik veriler ("diz_aci" ve "kalca_aci") ile hareketin benzetimi yapılmıştır. Daha sonra "joint sensor” blokları yardımıyla diz eklemi momenti (Moment_diz) hesaplanmıştır. Hesaplama işlemi ters dinamik yöntem [2] kullanılarak yapılmıştır. 
Burada eklemlerin kinematik değişimleri ve uzuvların kütle özellikleri bilindiğinden eklem momentleri hesaplanabilmiştir.

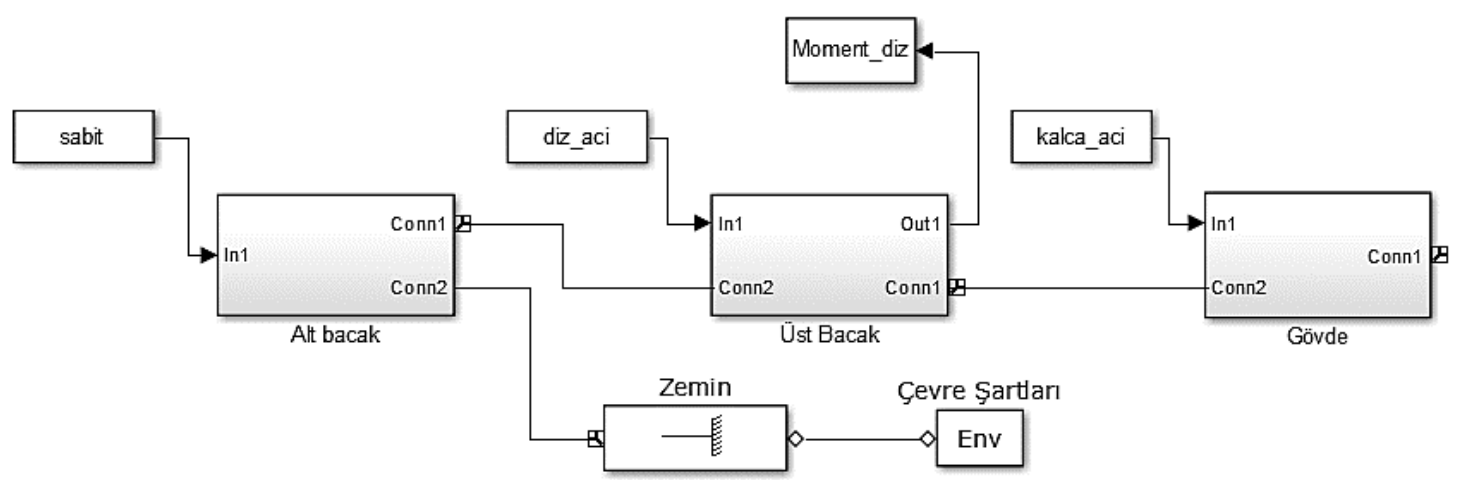

Şekil 2. Eklem-uzuv modelinin MATLAB Multibody yapısı

\subsection{Makine öğrenmesi algoritmaları}

Hareket analizi yardımıyla elde edilen kinematik veriler, uzuvların kütle özellikleri ve MATLAB benzetimi sonucunda hesaplanan diz eklemi moment verileri ile farklı makine öğrenmesi algoritmalarının eğitim, doğrulama ve test işlemleri gerçekleştirilmiştir. Tablo 1'de kullanılan veri setinden örnek bir kesit yer almaktadır. Burada açı; hareket sırasında değişen diz eklemi açısını, yük.; topuktan ayak bileğine kadar olan mesafeyi, kütle; alt bacak kütlesini, $\mathrm{I}_{\mathrm{xx}}-\mathrm{I}_{\mathrm{yy}}-\mathrm{I}_{\mathrm{zz}}$; üç farkl1 eksene göre atalet momentlerini, moment; diz eklemi momentini ifade etmektedir.

Tablo 1. Kullanılan veri setinden örnekler

\begin{tabular}{|c|l|c|c|c|c|c|c|c|}
\hline $\begin{array}{c}\text { Denek } \\
\text { No }\end{array}$ & $\begin{array}{c}\text { Zaman } \\
(\mathrm{s})\end{array}$ & $\begin{array}{c}\text { Aç1 } \\
(\mathrm{der})\end{array}$ & $\begin{array}{c}\text { Yük. } \\
(\mathrm{cm})\end{array}$ & $\begin{array}{c}\text { Kütle } \\
(\mathrm{kg})\end{array}$ & $\begin{array}{c}\mathrm{I}_{\mathrm{xx}} \\
\left(\mathrm{kg} \cdot \mathrm{m}^{2}\right)\end{array}$ & $\begin{array}{c}\mathrm{I}_{\mathrm{yy}} \\
\left(\mathrm{kg} \cdot \mathrm{m}^{2}\right)\end{array}$ & $\begin{array}{c}\mathrm{I}_{\mathrm{zz}} \\
\left(\mathrm{kg} \cdot \mathrm{m}^{2}\right)\end{array}$ & $\begin{array}{c}\text { Moment } \\
(\mathrm{Nm})\end{array}$ \\
\hline 1 & 0,297 & $-158,2$ & 50,4 & 5,8813 & 0,100215 & 0,100215 & 0,028116 & $-10,7031818$ \\
\hline 1 & 0,33 & $-157,8$ & 50,4 & 5,8813 & 0,100215 & 0,100215 & 0,028116 & $-13,3039344$ \\
\hline 1 & 0,363 & $-157,9$ & 50,4 & 5,8813 & 0,100215 & 0,100215 & 0,028116 & $-16,3668804$ \\
\hline 1 & 0,396 & $-155,9$ & 50,4 & 5,8813 & 0,100215 & 0,100215 & 0,028116 & $-19,4979187$ \\
\hline 1 & 0,429 & -156 & 50,4 & 5,8813 & 0,100215 & 0,100215 & 0,028116 & $-22,4503678$ \\
\hline
\end{tabular}

Verilerin tümüne bakıldığında toplamda 1227 adet veri satırı bulunduğu görülmektedir. Bu verilerin 1şı̆̆ıında Şekil 3' de görülen korelasyon 1sı haritası oluşturulmuştur. Isı haritasında da görüleceği üzere "açı" değişkeninin diz eklemi momenti ile negatif yönde yüksek korelasyon oluşturduğu anlaşılmaktadır. Bu bilgiler dikkate alınarak Şekil 4 'de görülen "açı" değişkenin analizi yapılmıştır. Buradaki negatiflik eklem momentinin yönüyle ilgilidir. 


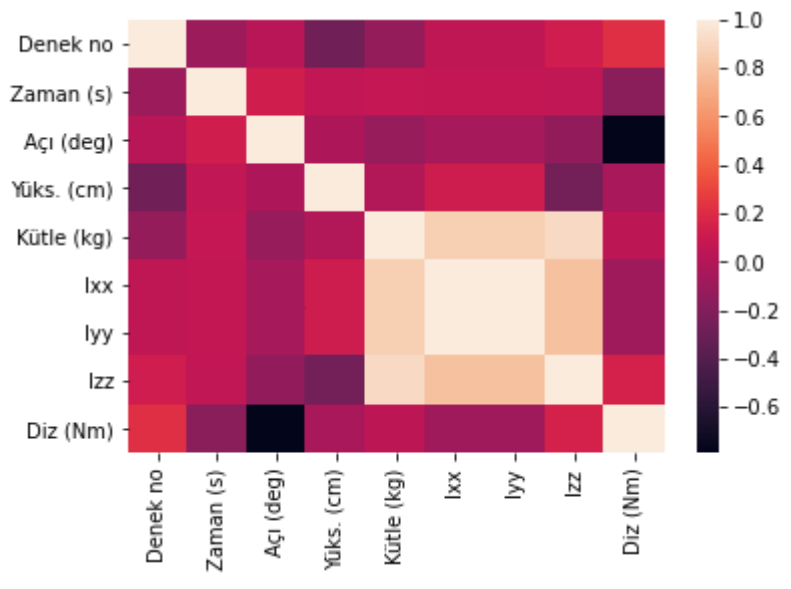

Şekil 3. Korelasyon 1sı haritası
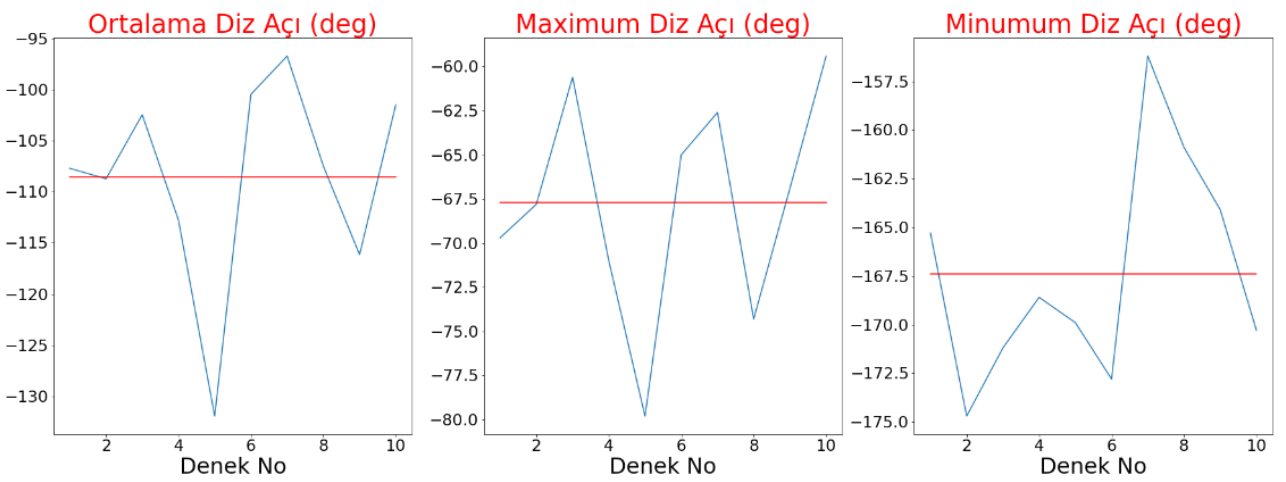

Şekil 4. Açı değişkeni analizi

\subsubsection{Doğrusal regresyon (DR}

İki veya daha fazla değişkenin, değişkenleri arasındaki ilişkilerin doğrusal şekilde ifade edilmesine dayanan algoritmadır. Bağımsız değiş̧ken kadar doğru oluşturulur. Bu çalışmada bağımsız değişkenler sırayla; zaman, açı, yük, kütle, Ixx, Izz katsayılar; -4.387 , -1.325, 5.152, -23.618, $960.462,3095.704$ ve sabit terim -326.333 olarak hesaplanmıştır.

\subsubsection{Destek vektör makineleri (DVM)}

Destek vektör makineleri (DVM), doğrusal regresyon modelinden farklı olarak belirli bir aralığa sahiptir. Bu aralık modelin hiper parametresi ile kontrol edilebilmektedir. Yapılan çalışmada varsayılan parametre değişkeni referans alınmıştır. Çekirdek hiper parametresi ise validasyon verileri ile yapılan testler ışığında doğrusal olarak seçilmiştir.

\subsubsection{Polinom regresyonu $(P R)$}

Polinom regresyon algoritmasında; bağımsız değişkenler ile bağımlı değişkenlerin arasında oluşturulan iliş̧i doğrusal değildir. Aradaki ilişki bir eğriyi temsil etmektedir. Eğrinin derecesi validasyon verisi üzerinde yapılan çalışmalar sonucu 2 olarak seçilmiştir. 


\subsubsection{Rassal orman (RO) algoritmast}

$\mathrm{Bu}$ yöntemde birden çok hiper parametresi bulunmaktadır. Bu çalışmada ağaç sayısı, hiper parametre validasyon verisi kullanılarak 50 adet alınmış olup, oluşturulacak maksimum dal sayısı 4 olarak seçilmiştir. Şekil 5'te rassal orman algoritması üzerinde geliştirdiğimiz modelin ağaç yapısı yer almaktadir.

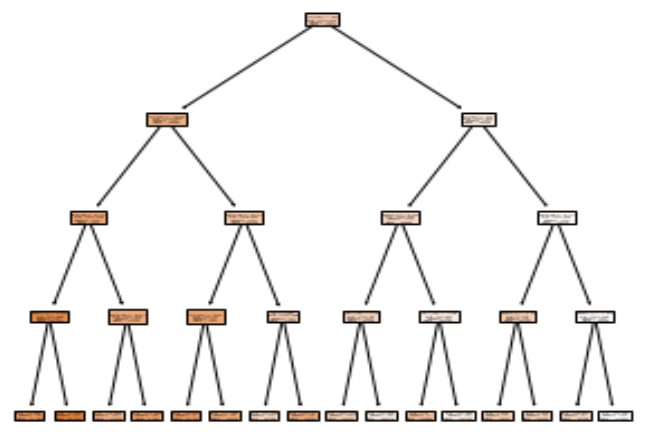

Şekil 5. Rassal ormanda oluşturulan 1.ağacın görseli

\subsubsection{XGBoost (XGB) algoritmast}

Bu çalışmada XGB algoritması ağaç tabanlı olduğundan rassal ormandaki hiper parametrelerin çoğuna sahiptir. Oluşturulan ağaç sayısı 50 ve maksimum dal sayısı 3 olarak seçilmiştir. XGB algoritması rassal ormandan faklı olarak gradian düşüş sağladığından öğrenme oranı hiper parametresine sahiptir. Öğrenme oranı validasyon verisi üzerinde yapılan denemeler sonunca bilinen en optimum değer olan 0.2 olarak seçilmiştir.

\subsubsection{Light GBM (LGM) algoritmast}

$\mathrm{Bu}$ yöntemde yaprak odaklı ağaç oluşturma sistemi olduğundan hata oranı azalır ve daha hızlı öğrenme gerçekleşir. Bu çalışmada oluşturulan ağaç sayısı 50 ve maksimum dal sayısı 3 olarak seçilmiş olup Xgboost algoritmasında olduğu gibi öğrenme oranına sahiptir. Öğrenme oranı çeşitli denemeler sonucunda bilinen en optimum sayı olan 0.1 olarak seçilmiştir.

\subsubsection{Derin yapay sinir ăğ (YSA)}

Bu çalışmada kullanılan derin YSA toplamda 3 katmandan oluşmakta olup bu katmanların 2 tanesi gizli katmandır. 3. katman ise çıkış katmanı olarak alınmıştır ve 1 adet nörondan oluşur. Katmanlar arasına aşırı öğrenmeyi önlemek için düğ̈̈m seyreltme yöntemi kullanılmıştır. Kayıp fonksiyonu olarak "Adam" seçilmiştir.

\section{Bulgular}

MATLAB Multibody araçları kullanılarak hesaplanan diz eklemi momenti Şekil 6'da yer almaktadır. Burada diz eklemi momentinin oturup kalkma hareketinin farklı evrelerinde $50 \mathrm{Nm}$ ile 
$-250 \mathrm{Nm}$ arasında değiştiği görülmektedir. Moment değerlerinde negatiflik sadece hareketin yönünü belirtmektedir. Vücut kitle indeksi (BMI) büyük olan deneklerde diz eklemi momenti belirgin bir şekilde yüksek çıkmaktadır.

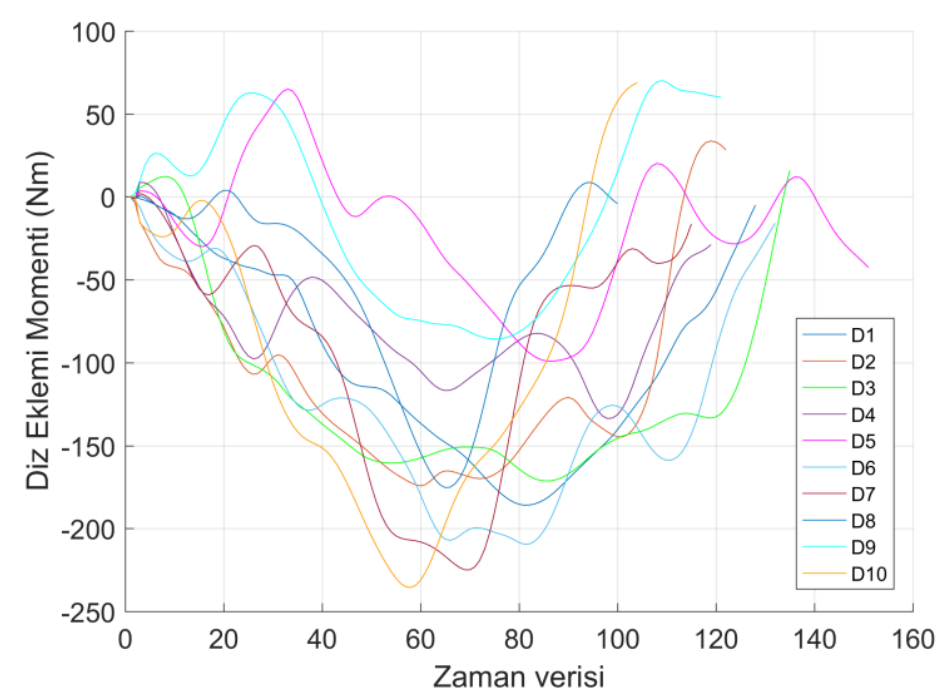

Şekil 6. Deneklerin oturup kalkma hareketi esnasındaki diz eklemi momentleri. D; denek.

Bu çalışmada bölüm 2.4'te bahsedilen 10 adet denekten elde edilen verilerin \% 10 validasyon verisi, kalan verinin $\% 15$ 'i test olarak kullanılmıştır. Test verisi üzerinde yapılan çalışmanın çıktıları ortalama kare hata yöntemi ile kıyaslanmış olup başarı oranları Tablo 2 'de gösterilmiştir. Yeni geliştirilen ağaç tabanlı XGB ve LightGBM algoritmaları tahmin başarısının, geleneksel yöntemlerden olan DR ve DVM'ye göre daha yüksek olduğu görülmektedir. Derin YSA, RO ve PR algoritmalarından elde edilen başarı oranlarının makul olduğu anlaşılmaktadır.

Tablo 2. Kullanılan algoritmaların test verisi üzerindeki başarı sonuçları

\begin{tabular}{|l|l|l|l|l|l|l|}
\hline DR & DVM & PR & RO & XGB & LGM & YSA \\
\hline$\% 75.005$ & $\% 71.589$ & $\% 83.459$ & $\% 85.086$ & $\% 94.079$ & $\% 89.542$ & $\% 82.089$ \\
\hline
\end{tabular}

Tablo 3'de farklı makine öğrenmesi algoritmalarının diz eklemi momentini tahminine yönelik sonuçları yer almaktadır. Veri bazında inceleme yapıldığında XGB algoritmasının en yüksek başarı oranına, DVM ve DR algoritmalarının ise en düşük başarı oranına sahip olduğu görülmektedir.

Tablo 3. Model tahminlerinin gerçek değerlerle karşılaştırılması

\begin{tabular}{|l|l|l|l|l|l|l|l|l|}
\hline Sira & Diz (Nm) & DR & DVM & RO & XGB & LGM & PR & YSA \\
\hline 0 & $-143,322$ & $-171,376$ & $-153,558$ & $-132,144$ & $-153,666$ & $-145,054$ & $-173,492$ & $-179,019$ \\
\hline 1 & $-103,512$ & $-106,643$ & $-111,418$ & $-98,2856$ & $-114,718$ & $-117,495$ & $-115,157$ & $-105,663$ \\
\hline 2 & $-154,404$ & $-178,982$ & $-159,699$ & $-173,681$ & $-167,37$ & $-169,532$ & $-175,392$ & $-170,497$ \\
\hline 3 & $-116,616$ & $-104,555$ & $-109,916$ & $-121,725$ & $-116,698$ & $-127,911$ & $-124,294$ & $-107,337$ \\
\hline 4 & $-133,382$ & $-44,6633$ & $-55,05$ & $-84,7604$ & $-99,6778$ & $-88,4348$ & $-75,7709$ & $-53,7508$ \\
\hline
\end{tabular}


Şekil 7 ise diz eklemi momenti gerçek değerleri ile farklı algoritmalar tarafindan hesaplanan moment değerlerinin dağılımını göstermektedir. Burada açık renkli noktalar gerçek moment değerinin, koyu renkli noktalar ise makine öğrenmesi algoritmaları tarafından tahmin edilen değerleri göstermektedir. Grafiklerde dikey eksen diz eklemi momentini $(\mathrm{Nm})$ yatay eksen ise zaman verisini ifade etmektedir.
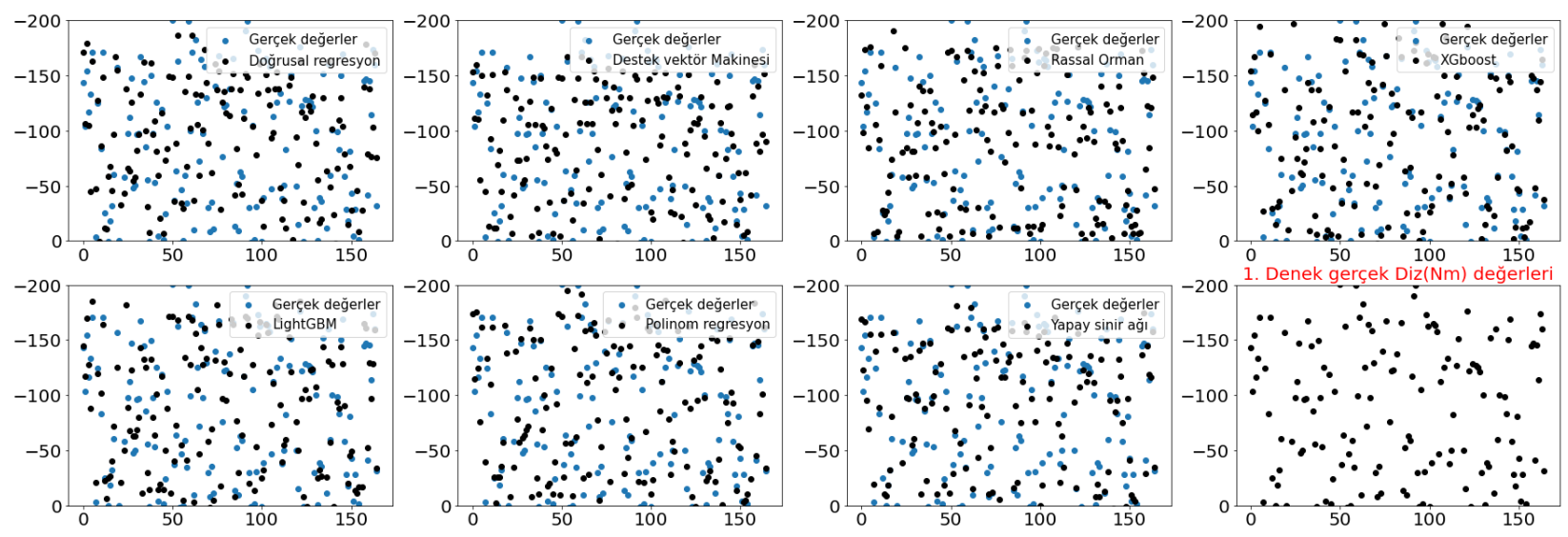

Şekil 7. Gerçek moment değerlerinin model tahminlerine göre dağılımı

\section{Tartışma}

Veriler üzerinde modelleme çalışması yapılmadan önce yy eksenindeki atalet momenti verisi çıkarılmıştır. xx ve yy eksenlerindeki atalet değerleri aynı olduğundan bu durum çoklu doğrusal bağlılığa sebep olmaktadır. Dolasıyla algoritmaların öğrenme başarısı düşmektedir. Şekil 3'de görüldüğü üzere öğrenme başarısındaki en büyük etki “açı” değişkenindedir.

Çalışma kapsamında yer alan denekler hem normal kilolu hem de aşırı kilolu kişilerden; hem uzun boylu hem de kısa boylu kişilerden seçildiğinden veriler arasında büyük farklılıklar bulunmaktadır. Oysaki literatüre bakıldığında yapay zeka teknikleri ile moment tahmininde benzer özellikte katılımcılar seçildiği görülmektedir [11, 12]. Farklı kütle ve atalet özelliklerine sahip kişiler üzerinde yapılan bu çalışmada bazı algoritmaların eklem momentlerini tahmin etmede daha düşük başarıya sahip olması beklenen bir durumdur. Örneğin 5 numaralı deneğin verileri ortalamadan büyük orada sapmaktadır. Bu durum algoritmaların başarısını düşürmektedir. Bu sorun 5 numaralı deneğe benzer özelliklere sahip ilave denekler eklenerek veya korelasyonu "açı" değişkeninden daha yüksek bir bağımsız değişken ilave edilerek aşılabilir.

Veri setinin diz eklemi moment dağılımın doğrusal bir şekilde ilerlememesi geleneksel yöntemler olan DR ve DVM algoritmasının başarı oranın diğer algoritmalara göre düşük kalmasına sebep olmuştur. YSA, XGB ve LGM algoritmalarının başarısı hiperparemetlerin validasyon veri setinde üzerinde farklı şekillerde denenmesiyle arttırılabilir. 


\section{Sonuç}

$\mathrm{Bu}$ çalışmada diz eklemi momenti, kütle ve atalet özelliklerine dayalı veriler kullanılarak farklı makine öğrenmesi algoritmaları ile tahmin edilmiştir. Burada en yüksek başarı XGB algoritması kullanılarak elde edilmiştir. Oturup kalkma hareketinin analizine yönelik geliştirilen bu yöntemler yürüyüş, üst ekstremite, egzersiz vb. hareketlere de uyarlanabilir. Böylece farklı uygulamalar için gerekli eklem momentleri benzer şekilde tahmin edilebilir.

\section{References}

[1] De Looze MP, Kingma I, Bussmann JBJ, Toussaint HM. Validation of a dynamic linked segment model to calculate joint moments in lifting. Clinical Biomechanics 1992;7:161-9.

[2] Silva MPT, Ambrosio JAC. Kinematic data consistency in the inverse dynamic analysis of biomechanical systems. Multibody System Dynamics 2002;8:219-39.

[3] Trinler U, Schwameder H, Baker R, Alexander N. Muscle force estimation in clinical gait analysis using AnyBody and OpenSim. Journal of Biomechanics 2019;86:55-63.

[4] Ozada N. Biomechanical model of knee collateral ligament injury with six degrees of freedom. Medical \& Biological Engineering \& Computing 2016:54:821-30.

[5] Cilli M, Serbest K, Kayaoglu E. The effect of body weight on joint torques in teenagers: Investigation of sit-to-stand movement. Clinical Biomechanics 2021;83:105288.

[6] Atasoy A, Topasş E, Kuchimov S, Gulfize S, Turpcu M, Kaplanoglu E, et al. Biomechanical design of an anthropomorphic prosthetic hand. 7th IEEE International Conference on Biomedical Robotics and Biomechatronics (Biorob) 2018;732-6.

[7] Mouloodi S, Rahmanpanah H, Gohari S, Burvill C, Tse KM, Davies HM. What can artificial intelligence and machine learning tell us? A review of applications to equine biomechanical research. Journal of the Mechanical Behavior of Biomedical Materials 2021;104728.

[8] Jia G, Lam HK, Liao J, Wang R. Classification of electromyographic hand gesture signals using machine learning techniques. Neurocomputing 2020;401:236-48.

[9] Yeadon MR. The simulation of aerial movement-II. A mathematical inertia model of the human body. Journal of Biomechanics 1990;23:67-74.

[10] Brown D. Tracker Video Analysis and Modeling Tool. 2009. (Computer Software).

[11] Xiong B, Zeng N, Li H, Yang Y, Li Y, Huang M, et al. Intelligent prediction of human lower extremity joint moment: an artificial neural network approach. IEEE Access 2019;7: 29973-80.

[12] Zhang L, Li Z, Hu Y, Smith C, Farewik EMG, Wang R. Ankle joint torque estimation using an emg-driven neuromusculoskeletal model and an artificial neural network model. IEEE Transactions on Automation Science and Engineering 2020;18:564-73. 